Volume 129, Number 8, Pages 2473-2481

S 0002-9939(00)05796-8

Article electronically published on December 7, 2000

\title{
ANALYTIC LINEARIZABILITY OF SOME RESONANT VECTOR FIELDS
}

\author{
J. BASTO-GONÇALVES AND I. CRUZ
}

(Communicated by Carmen Chicone)

\begin{abstract}
A method allowing the linearization of vector fields with resonant eigenvalues is presented, the admissible nonlinearities being characterized by conditions that are easy to check.
\end{abstract}

Let $X(x, w)$ be a vector field on a domain $U$ in $\mathbf{C}^{n}$ depending on parameters $w$ in the domain $W \subset \mathbf{C}^{m} ; X$ is assumed to be holomorphic as a map $X: U \times W \longrightarrow \mathbf{C}^{n}$.

The vector field $X$ is supposed to have a singular point at the origin in $\mathbf{C}^{n}$ with, for the moment, linear part $A$ independent of $w$ :

$$
X(x, w)=A x+a(x, w), \quad a(0, w)=\frac{\partial a}{\partial x}(0, w)=0 .
$$

$X$ is said to be biholomorphically equivalent to its linear part if there exists a holomorphic change of coordinates $y=\psi(x, w)$, depending on the parameters $w$, preserving the origin, $\psi(0, w)=0$, with inverse (as a function only of $x) x=\xi(y, w)$ also holomorphic, such that in the new coordinates the nonlinear part is zero:

$$
\frac{\partial \xi}{\partial x}(\psi(y, w), w) X(\psi(y, w), w)=A y .
$$

Let $\lambda=\left(\lambda_{1}, \ldots, \lambda_{n}\right) \in \mathbf{C}^{n}$ be the vector of the eigenvalues of the linear part $A$ of $X$, which are not assumed to be distinct (see [1]).

The eigenvalues are said to be resonant of order $k$ if there exists $m=\left(m_{1}, \ldots, m_{n}\right)$, with $m_{i}$ nonnegative integers and $|m|=m_{1}+\cdots+m_{n}=k \geq 2$, such that for some $s$ :

$$
\lambda_{s}=m \cdot \lambda .
$$

The vector $\lambda$ belongs to the Poincaré domain if zero is not in the convex hull of the $n$ points $\left\{\lambda_{1}, \ldots, \lambda_{n}\right\}$ in the complex plane, and to the Siegel domain if zero is in the interior of that set.

If we think of $X$ as a vector field in $U \times W \subset \mathbf{C}^{n+m}$ with null component along $\mathbf{C}^{m}$, then we have resonances on the linear part of $X$ and the theorems of Poincaré and Siegel on the linearization of vector fields (see [1]) cannot be used directly. The version of those theorems including parameters below follows from the very general

Received by the editors August 16, 1999 and, in revised form, December 7, 1999.

1991 Mathematics Subject Classification. Primary 58F36, 32S65, 34A20, 34A34.

The first author's research was supported by JNICT, and by the Calouste Gulbenkian Foundation.

The second author's research was supported by JNICT. 
results in [3]. The analogous situation for the $C^{\infty}$ case was solved by S. Sternberg [9], and later R. Roussarie [8] proved a version with parameters.

Theorem 1. Poincaré theorem with parameters: If the vector $\lambda$ belongs to the Poincaré domain and is nonresonant, $X$ is biholomorphically equivalent to its linear part in the neighbourhood of the singular point.

The vector $\lambda$ is said to be of type $(C, \nu)$ if, for any $s$ :

$$
\left|\lambda_{s}-\lambda \cdot m\right| \geq \frac{C}{|m|^{\nu}}
$$

with $m=\left(m_{1}, \ldots, m_{n}\right), m_{i}$ nonnegative integers and $|m| \geq 2$.

Clearly if $\lambda$ is of type $(C, \nu)$, then it is nonresonant. If $\lambda$ belongs to the Poincaré domain and is nonresonant, then it is of type $(C, \nu)$ for convenient $C$ and $\nu$. Every point in the Poincaré domain satisfies not more than a finite number of resonances and has a neighbourhood where no other resonance relation is satisfied [1].

Theorem 2. Siegel theorem with parameters: If $\lambda$ is of type $(C, \nu), X$ is biholomorphically equivalent to its linear part in the neighbourhood of the singular point.

Our aim is to obtain linearization results when there are resonances; this forces us to restrict the nonlinear terms. The linearizability of $X$ at $x_{0}$ is not determined by its 1-jet (linear part), but the allowed nonlinearities are characterized by conditions that are easy to check.

In this context, $k$-determinacy means that the conditions involve only the $k$ jet of the vector field. Whenever the eigenvalues are in the Poincaré domain the linearization problem is finitely determined.

The analytic linearizability of real analytic vector fields with all eigenvalues equal and nonzero, therefore nonresonant, and depending on one parameter, was proved in 4. Here we prove that the same method can be used for complex holomorphic vector fields depending on parameters for a much larger class of eigenvalues, in particular allowing resonances. An application of these results is discussed in [2].

All results are also valid in the real analytic category, and applicable to maps instead of vector fields, with standard adaptations.

\section{Holomorphic Linearizability of SOME RESONANT VECTOR FIELDS}

Again let $X(x, w)$ be a holomorphic vector field on a domain $U$ in $\mathbf{C}^{n}$ depending on parameters $w$ in the domain $W \subset \mathbf{C}^{m}$, with a singular point at $0 \in \mathbf{C}^{n}$ :

$$
X(x, w)=A(w) x+a(x, w), \quad a(0, w)=\frac{\partial a}{\partial x}(0, w)=0 .
$$

Let $\lambda(w)=\left(\lambda_{1}(w), \ldots, \lambda_{n}(w)\right) \in \mathbf{C}^{n}$ be the vector of the eigenvalues of the linear part $A(w)$ of $X$. If

$$
a(x, w)=\sum_{i=1}^{n} \sum_{|I| \geq 2,|J| \geq 0} a_{I J}^{i} x^{I} w^{J} e_{i},
$$

then the monomial $x^{I} w^{J} e_{i}$ is said to be resonant if $I \cdot \lambda-\lambda_{i}=0$ for some $w \in W$.

The Poincaré-Dulac theorem [1] allows the elimination of nonresonant terms by a formal change of variables, holomorphic under certain conditions, when in fact $A(w)$ does not depend on $w$. It does not guarantee that the linearization can be 
performed if the nonlinearity $a$ does not contain any resonant term, as the following example shows:

Example 1. Let $X(x, y)=\left(-x+y^{3}, y+x^{4} y\right)$ be a vector field in $\mathbf{C}^{2}$. The eigenvalues are -1 and 1 , therefore resonant, but the nonlinearity does not contain resonant monomials of the form $x^{k+1} y^{k} e_{1}$ or $x^{k} y^{k+1} e_{2}$.

The first step in the Poincaré-Dulac method leads to the change of variables $\xi=x-y^{3} / 4, \eta=y$, eliminating the lower order term of the nonlinearity, but in the new coordinates

$$
X(\xi, \eta)=\left(-\xi-\frac{3}{4}\left(\xi+\frac{\eta^{3}}{4}\right)^{4} \eta^{3}, \eta+\left(\xi+\frac{\eta^{3}}{4}\right)^{4} \eta\right)
$$

and the resulting nonlinearity now has resonant monomials $\xi^{4} \eta^{3} e_{1}$ and $\xi^{3} \eta^{4} e_{2}$.

Theorem 3. Let $X$ be a holomorphic vector field on a neighbourhood $U \times W$ of the origin in $\mathbf{C}^{n+m}$ which, in coordinates $(z, w)$, can be written as

$$
X(x, w)=A(w) x+a(x, w), \quad A(w)=\operatorname{diag}\left\{\lambda_{1}(w), \ldots, \lambda_{n}(w)\right\}
$$

If the nonlinearity $a(x, w)$ is admissible, i.e. all $I \cdot \lambda-\lambda_{i}$ are positive integer multiples of a fixed complex number for every monomial $x^{I} w^{J} e_{i}$ in a and $w \in W$, there exists an holomorphic change of coordinates $y=\psi(x, w)$ linearizing $X$.

Remark 1 . In the real analytic category, the nonlinearity $a(x, w)$ is admissible if all $I \cdot \lambda-\lambda_{i}$ are positive integer multiples of a fixed real number for every monomial $x^{I} w^{J} e_{i}$ in $a$ and $w \in W$. Also the real canonical form is assumed for $A$ instead of the canonical Jordan form, all blocks being one dimensional if corresponding to real eigenvalues and two dimensional if the eigenvalues are complex. $a(x, w)$ admissible means, in particular, that the components of $a$ corresponding to complex eigenvalues are zero, and therefore are not relevant to the linearization procedure. Thus $A$ can be assumed to be diagonal with all eigenvalues real, and the proof of the linearization in the real analytic category is identical to the one in the complex case.

Proof. Without loss of generality it may be assumed that all $I \cdot \lambda-\lambda_{i}$ are positive integers independent of $w$, since a change of coordinates that linearizes a vector field also linearizes any multiple of it.

Suppose that $\psi$ exists and let $\xi(y, w)$ denote its inverse (as a function of $x$ alone). Then $\xi$ will satisfy the system of partial differential equations

$$
\lambda_{1} y_{1} \frac{\partial \xi_{i}}{\partial y_{1}}+\cdots+\lambda_{n} y_{n} \frac{\partial \xi_{i}}{\partial y_{n}}=\lambda_{i} \xi_{i}+a_{i}(\xi, w), \quad i=1, \ldots, n
$$

If the initial value problem composed of (1) and the following initial conditions:

$$
\xi(0, w)=0, \quad \frac{\partial \xi}{\partial y}(0, w) \text { nonsingular }
$$

has a solution $\xi(z, w)$ which is holomorphic at $0 \in \mathbf{C}^{n+m}$ the proof of the theorem is finished, since (2) implies that $\xi$ is locally invertible, as a function of $y$ alone. 
1.1. Change of variables. Let $z=\left(z_{1}, \ldots, z_{n}\right)$ be defined by

$$
y_{1}=z_{1}^{\lambda_{1}}, \quad y_{i}=z_{1}^{\lambda_{i}} z_{i}
$$

and let $\zeta$ be the expression of $\xi$ in the new variables $z$. Then

$$
\begin{aligned}
\zeta(z, w) & =\xi\left(z_{1}^{\lambda_{1}}, z_{1}^{\lambda_{2}} z_{2}, \ldots, z_{1}^{\lambda_{n}} z_{n}, w\right), \\
\frac{\partial \zeta}{\partial z_{1}} & =\lambda_{1} z_{1}^{\lambda_{1}-1} \frac{\partial \xi}{\partial y_{1}}+\sum_{j=2}^{n} \lambda_{j} z_{1}^{\lambda_{j}-1} z_{j} \frac{\partial \xi}{\partial y_{j}},
\end{aligned}
$$

and (11) can be rewritten as

$$
z_{1} \frac{\partial \zeta_{i}}{\partial z_{1}}=\lambda_{i} \zeta_{i}+a_{i}(\zeta, w), \quad i=1, \ldots, n .
$$

Introduce new unknown functions $\eta$ by

$$
\zeta_{i}=z_{1}^{\lambda_{i}} \eta_{i}, \quad i=1, \ldots, n .
$$

Then

$$
\begin{aligned}
\frac{\partial \zeta_{i}}{\partial z_{1}} & =\lambda_{i} z_{1}^{\lambda_{i}-1} \eta_{i}+z_{1}^{\lambda_{i}} \frac{\partial \eta_{i}}{\partial z_{1}}, \\
z_{1} \frac{\partial \zeta_{i}}{\partial z_{1}} & =\lambda_{i} \zeta_{i}+z_{1}^{\lambda_{i}+1} \frac{\partial \eta_{i}}{\partial z_{1}} \\
& =\lambda_{i} \zeta_{i}+a_{i}(\zeta, w), \quad i=1, \ldots, n,
\end{aligned}
$$

and therefore (3) is equivalent to

$$
z_{1}^{\lambda_{i}+1} \frac{\partial \eta_{i}}{\partial z_{1}}=a_{i}\left(z_{1}^{\lambda} \eta, w\right), \quad \text { with } z_{1}^{\lambda} \eta=\left(z_{1}^{\lambda_{1}} \eta_{1}, \ldots, z_{1}^{\lambda_{n}} \eta_{n}\right), \quad i=1, \ldots, n .
$$

1.2. Reduction to an ordinary differential equation. Thus the system of partial differential equations (11) can be written as a system of nonautonomous ordinary differential equations

$$
\frac{d}{d z_{1}} \eta_{i}=\alpha_{i}\left(z_{1}, \eta, w\right), \quad i=1, \ldots, n,
$$

depending on parameters $w$. That $\alpha$ is holomorphic around the origin is an immediate consequence of $a$ being admissible.

Writing $a_{i}(x, w)$ as an absolutely convergent power series we have

$$
a_{i}(x, w)=\sum_{|I|=2,|J|=0}^{\infty} a_{I J}^{i} x^{I} w^{J} .
$$

Then

$$
\alpha_{i}\left(z_{1}, \eta, w\right)=\sum_{|I|=2,|J|=0}^{\infty} a_{I J}^{i} z_{1}^{I \cdot \lambda-\lambda_{i}-1} \eta^{I} w^{J},
$$

which converges for $z_{1}=1$ and $|(\eta, w)|$ small enough.

Of course this system was obtained using changes of coordinates that are not valid in a full neighbourhood of the origin, but nevertheless this system is well defined even at $z_{1}=0$.

From the existence and uniqueness theorem for ordinary differential equations in the complex domain (7), system (4) has a solution which is holomorphic as a function of $z_{1}$, the parameters and also the initial conditions. It will be shown that it is possible to select the initial conditions so that the map $\xi$, corresponding to $\eta$, 
is holomorphic in a neighbourhood of $(y, w)=(0,0)$ and a solution of the initial value problem (11), (2).

So let $\eta(z, w)$ be the solution of (4) with initial conditions

$$
\eta(0, \hat{z}, w)=(1, \hat{z}), \quad \hat{z}=\left(z_{2}, \ldots, z_{n}\right) .
$$

Assume for the moment that the corresponding $\xi$ is holomorphic. Then $\xi$ satisfies

$$
\xi_{i}\left(z_{1}^{\lambda_{1}}, z_{1}^{\lambda_{2}} z_{2}, \ldots, z_{1}^{\lambda_{n}} z_{n}, w\right)=z_{1}^{\lambda_{i}} \eta_{i}(z, w), \quad i=1 \ldots, n,
$$

and so $\xi(0, w)=0$. Taking the derivative with respect to $z_{1}$,

$$
\lambda_{1} z_{1}^{\lambda_{1}-1} \frac{\partial \xi_{i}}{\partial y_{1}}+\sum_{j=2}^{n} \lambda_{j} z_{1}^{\lambda_{j}-1} z_{j} \frac{\partial \xi_{i}}{\partial y_{j}}=\lambda_{i} z_{1}^{\lambda_{i}-1} \eta_{i}+z_{1}^{\lambda_{i}} \frac{\partial \eta_{i}}{\partial z_{1}}
$$

multiplying by $z_{1}$ and writing in terms of the $y$ variables, we get

$$
\lambda_{1} y_{1} \frac{\partial \xi_{i}}{\partial y_{1}}+\sum_{j=2}^{n} \lambda_{j} y_{j} \frac{\partial \xi_{i}}{\partial y_{j}}=\lambda_{i} z_{1}^{\lambda_{i}} \eta_{i}+z_{1}^{\lambda_{i}+1} \frac{\partial \eta_{i}}{\partial z_{1}}=\lambda_{i} \xi_{i}+a_{i}
$$

which show that $\xi$ is a solution of the partial differential equations (11).

1.3. Conditions for the holomorphy of $\xi$. Writing $\eta_{i}(z, w)$ as an absolutely convergent power series

$$
\eta_{i}(z, w)=\sum_{|I|,|J|=0}^{\infty} b_{I J}^{i} z^{I} w^{J}
$$

and taking $\hat{I}=\left(i_{2}, \ldots, i_{n}\right),|\hat{I}|=i_{2}+\ldots+i_{n}$ and $\hat{z}^{\hat{I}}=z_{2}^{i_{2}} \ldots z_{n}^{i_{n}}$, it then follows from (6) that $\xi_{i}(y, w)$ is formally represented by the series

$$
\sum_{|I|,|J|=0}^{\infty} b_{I J}^{i} y_{1}^{\left(i_{1}-\hat{I} \cdot \hat{\lambda}+\lambda_{i}\right) / \lambda_{1}} \hat{y}^{\hat{I}} w^{J} .
$$

A monomial $z^{I} w^{J} e_{i}$ is said to satisfy condition $\mathrm{H}$ if there exists a nonnegative integer $k$ such that

$$
i_{1}=\hat{I} \cdot \hat{\lambda}-\lambda_{i}+k \lambda_{1}
$$

A holomorphic map is said to satisfy condition $H$ if all monomials with nonzero coefficients in its power series centered at the origin satisfy that condition.

A necessary condition for $\xi_{i}$ to be holomorphic is that $\eta$ satisfies condition $\mathrm{H}$. In fact this is also a sufficient condition.

Lemma 1. Suppose that $\sum_{|I|,|J|=0}^{\infty} b_{I J}^{i} z^{I} w^{J}$ converges absolutely in the disc $D_{r}^{n+m}$ for all $i=1, \ldots, n$, and condition $H$ is verified. Then $\xi$ is a holomorphic map in a neighbourhood of the origin in $\mathbf{C}^{n+m}$.

Proof. From the Cauchy inequalities there exists $C \in \mathbf{R}^{+}$such that

$$
\left|b_{I J}^{i}\right| \leq \frac{C}{r^{|I|+|J|}}
$$

Since condition $\mathrm{H}$ holds, $\xi_{i}(y, w)$ is represented by the formal power series

$$
\xi_{i}(y, w)=\sum_{k,|\hat{I}|,|J|=0}^{\infty} b_{I J}^{i} y_{1}^{k} \hat{y}^{\hat{I}} w^{J}
$$


with $i_{1}=\hat{I} \cdot \hat{\lambda}-\lambda_{i}+k \lambda_{1} \geq 0$. Then

$$
\sum_{k,|\hat{I}|,|J|=0}^{\infty}\left|b_{I J}^{i} y_{1}^{k} \hat{y}^{\hat{I}} w^{J}\right| \leq C \sum_{k,|\hat{I}|,|J|=0}^{\infty} \frac{\left|y_{1}^{\left(i_{1}-\hat{I} \cdot \hat{\lambda}+\lambda_{i}\right) / \lambda_{1}} \hat{y}^{\hat{I}} w^{J}\right|}{r^{|I|+|J|}}
$$

and this last series is convergent if

$$
\left|y_{1}\right|<r^{\lambda_{1}}, \quad\left|y_{i}\right|<r^{\lambda_{i}+1}, i>1, \quad|w|<r .
$$

These conditions define a neighbourhood $\mathcal{N}$ of the origin in $\mathbf{C}^{n+m}$ where the formal power series corresponding to all $\xi_{i}$ are absolutely convergent. Then $\xi$ is holomorphic in $\mathcal{N}$.

1.4. Holomorphy of $\xi$. That condition $\mathrm{H}$ is verified will be proved by induction on the order in $z_{1}$ of the terms in the power series of $\eta$.

From the initial conditions (5) it follows that

$$
\eta_{1}(z, w)=1+z_{1} R_{1,1}(z, w), \quad \eta_{i}(z, w)=z_{i}+z_{1} R_{i, 1}(z, w), i>1,
$$

and therefore the terms of order zero in $z_{1}$ verify condition $\mathrm{H}: 1=z^{I}$, with $I=0$ and $k=1$, and similarly $z_{i}=z^{I}$ with $i_{j}=0$ for all $j$ except $i_{i}=1$, and $k=0$.

Now assume that condition $\mathrm{H}$ is verified up to order $l$, that is, $\eta_{i}$ can be written as $\eta_{i}=\eta_{i}^{l}+z_{1}^{l+1} R_{i, l+1}(z, w)$, where

$$
\eta_{i}^{l}=\sum_{r=0}^{l} \sum_{\hat{I}, J} b_{r \hat{I} J}^{i} z_{1}^{r} \hat{z}^{\hat{I}} w^{J}, \quad r=\hat{I} \cdot \hat{\lambda}-\lambda_{i}+k \lambda_{1} \text { for some } k .
$$

The differential equations (4) can be written as

$$
z_{1} \frac{d}{d z_{1}} \eta_{i}=z_{1}^{-\lambda_{i}} a_{i}\left(z_{1}^{\lambda} \eta, w\right), \quad i=1, \ldots, n,
$$

and so, as the monomials appearing (i.e. with possibly nonzero coefficients) in $\eta_{i}$ are exactly the same as the ones appearing in $z_{1} d \eta_{i} / d z_{1}$, again, if

$$
a_{i}(x, w)=\sum_{|I|=2,|J|=0}^{\infty} a_{I J}^{i} x^{I} w^{J},
$$

then

$$
z_{1}^{-\lambda_{i}} a_{i}\left(z_{1}^{\lambda} \eta, w\right)=\sum_{|I|=2,|J|=0}^{\infty} a_{I J}^{i} z_{1}^{I \cdot \lambda-\lambda_{i}} \eta^{I} w^{J}
$$

and $z_{1}^{-\lambda_{i}} a_{i}$ has no terms of order less than 1 in $z_{1}$. The monomials in $\eta$ up to order $l+1$ in $z_{1}$ can be determined from the knowledge of the ones up to order $l$ through equation (7): they have to appear in

$$
z_{1}^{-\lambda_{i}} a_{i}\left(z_{1}^{\lambda} \eta^{l}, w\right)=z_{1}^{-\lambda_{i}} \sum a_{I J}^{i}\left(z_{1}^{\lambda} \eta^{l}\right)^{I} w^{J} .
$$

All these monomials have the form

$$
z_{1}^{-\lambda_{i}}\left(z_{1}^{\lambda_{1}} \mathcal{M}_{1}\right)^{i_{1}} \ldots\left(z_{1}^{\lambda_{n}} \mathcal{M}_{n}\right)^{i_{n}} w^{J}
$$

where $\mathcal{M}_{i} e_{i}$ denotes monomials of $\eta_{i}^{l}$, therefore satisfying condition $\mathrm{H}$. 
If $z^{I} w^{J} e_{i}$ and $z^{K} w^{L} e_{j}$ satisfy condition $\mathrm{H}$, then so does the monomial

$$
z_{1}^{-\lambda_{k}} z_{1}^{\lambda_{i}} z^{I} w^{J} z_{1}^{\lambda_{j}} z^{K} w^{L} e_{k}
$$

and, by induction, all the products in (8) satisfy condition $\mathrm{H}$, and $\xi$ is holomorphic.

1.5. Biholomorphy of $\xi$. The partial derivative of $\xi_{i}$ with respect to $y_{1}$ at the origin is the coefficient of $y_{1} e_{i}$ in the series development of $\xi$. As $y_{1} e_{i}=y^{I} w^{J} e_{i}$, with $I=e_{1}$ and $J=0$, the corresponding monomials in $\eta$ are $1 e_{1}$, if $i=1$, and $z_{1}^{\lambda_{1}-\lambda_{i}} e_{i}$, if $i>1$.

If $\lambda_{1}=\lambda_{i}$ with $i>1$, the coefficient of $z_{1}^{\lambda_{1}-\lambda_{i}} e_{i}=e_{i}$ is zero, since $\eta_{i}=$ $z_{i}+z_{1} R_{i, 1}(z, w)$; otherwise $z_{1}^{\lambda_{1}-\lambda_{i}} e_{i}$ has to be a product of the form

$$
z_{1}^{-\lambda_{i}}\left(z_{1}^{\lambda_{1}} \mathcal{M}_{1}\right)^{i_{1}} \ldots\left(z_{1}^{\lambda_{n}} \mathcal{M}_{n}\right)^{i_{n}}
$$

with $|I|$ at least 2, as explained before. More precisely,

$$
z_{1}^{\lambda_{1}-\lambda_{i}}=z_{1}^{-\lambda_{i}}\left(z_{1}^{\lambda_{1}} z_{1}^{k_{1} \lambda_{1}-\lambda_{1}}\right)^{i_{1}} \ldots\left(z_{1}^{\lambda_{n}} z_{1}^{k_{n} \lambda_{1}-\lambda_{n}}\right)^{i_{n}}=z_{1}^{(k \cdot I) \lambda_{1}-\lambda_{i}} .
$$

Since $z_{1}^{k_{j} \lambda_{1}-\lambda_{j}}$ has zero coefficient if $k_{j}=0$, it is necessary that $k \cdot I=1$ with $|I| \geq 2$ and all $k_{j}$ positive.

As this is impossible, the coefficient of $z_{1}^{\lambda_{1}-\lambda_{i}} e_{i}$ has to be zero, and thus

$$
\frac{\partial \xi_{1}}{\partial y_{1}}(0, w)=1, \quad \frac{\partial \xi_{i}}{\partial y_{1}}(0, w)=0 i>1 .
$$

The reasoning for the other partial derivatives is similar, leading to a Jacobian at $(0, w)$ equal to the identity. Thus $\xi$ is a biholomorphic change of coordinates, and the proof of Theorem 3 is complete.

Example 2. Let $X(x)$ be a vector field in $\mathbf{R}^{4}$ :

$$
X(x)=A x+a(x), \quad A x=\left(x_{1},-x_{2}, x_{3},-x_{4}\right) .
$$

As the eigenvalues $\lambda=(1,-1,1,-1)$ are resonant and the number of resonant monomials is not finite, classical results can only guarantee the existence of a formal change of coordinates to a normal form containing only resonant monomials besides the linear part.

When the nonlinearity $a$ is admissible (this is independent of parameters), Theorem 3 guarantees the existence of an analytic change of coordinates that linearizes $X(x)=A x+a(x)$. As the proof is constructive, it provides a method for the computation of that change of coordinates. For example, the vector field $X(x)=\left(x_{1}+x_{1}^{2} x_{2} x_{3},-x_{2}, x_{3},-x_{4}+x_{2} x_{3}\right)$ is linearized by

$$
\xi(y)=\left(\frac{y_{1}}{1-y_{1} y_{2} y_{3}}, y_{2}, y_{3}, y_{4}+y_{2} y_{3}\right) .
$$

\section{Finite Determinacy}

Recall that the nonlinearity $a(x, w)$ is admissible, in the context of real analytic vector fields, if $I \cdot \lambda-\lambda_{i}$ are all positive (or all negative) integer multiples of a real number for every monomial $x^{I} t^{J} e_{i}$ in $a_{i}(x, w)$.

Let $\sigma$ be the ratio

$$
\sigma=\frac{\max \left|\Re \lambda_{i}\right|}{\min \left|\Re \lambda_{i}\right|}
$$

and denote by $d$ the biggest integer not greater than $\sigma, d=[\sigma]$. 
When considering the linearization of holomorphic vector fields with resonant eigenvalues in the Poincaré domain, the linearization procedure of section 1 allows $j^{d}(X)(x, w)=A x+$ admissible term, instead of just $j^{d}(X)(x, w)=A x$, as it is necessary to use the Poincaré-Dulac theorem.

Theorem 4. Let $X$ be an analytic vector field with the eigenvalues vector $\lambda$ in the Poincaré domain, and

$$
j^{d}(X)(x, w)=A x+\text { admissible term } .
$$

Then $X$ is analytically linearizable.

Proof. The linearization procedure of section 1 works for $j^{d}(X)(x, w)=A x+$ admissible term, and gives a change of coordinates linearizing $j^{d}(X)(x, w)$. Making that change of coordinates in $X$ results in a new vector field $Y(x, w)=A x+$ nonlinear part.

All monomials in the nonlinear part of $Y$ are of the type $x^{I} w^{j}$ with $|I|>\sigma$. Now it follows from the Poincaré-Dulac theorem that $Y$, and therefore $X$, can be linearized.

\section{Applications}

An interesting application of Theorems 3 and 4 is the linearization of Poisson structures in the analytic category (see 6] for the original $C^{\infty}$ result and [2] for the analytic case with resonances). In such applications $X$ is a Hamiltonian vector field associated with the Poisson structure $P$, whose linearizability allows (under some restrictions) the linearization of the original Poisson structure. One of these restrictions is precisely that the linear part of such a vector field must be independent of the parameters.

Example 3. Consider the family of Poisson tensors given by

$$
\begin{aligned}
P= & \left(-x+t^{n} x y^{m}\right) \frac{\partial}{\partial x} \wedge \frac{\partial}{\partial t}+\left(y+t^{n} y^{m+1}\right) \frac{\partial}{\partial y} \wedge \frac{\partial}{\partial t} \\
& +c(t) x y\left(1+t^{n} y^{m}\right) \frac{\partial}{\partial x} \wedge \frac{\partial}{\partial y},
\end{aligned}
$$

where $n$ and $m$ are integers $(n \geq 0$ and $m \geq 1)$, and $c(t)$ is any analytic function of $t$. The vector field under consideration is the Hamiltonian vector field of the coordinate $t$, i.e.,

$$
X=\left(-x+t^{n} x y^{m}\right) \frac{\partial}{\partial x}+\left(y+t^{n} y^{m+1}\right) \frac{\partial}{\partial y} .
$$

The linear part of $X$ is given by $A=\left(\begin{array}{rr}-1 & 0 \\ 0 & 1\end{array}\right)$, i.e., $A$ is resonant and its eigenvalues do not belong to the Poincaré domain. Theorem [3 implies that $X$ is analytically linearizable, and it is possible to show that the same happens with the above family $P$ (see [2] for the details). Furthermore, we can produce an explicit formula for the linearizing change of coordinates, which is

$$
(x, y) \longmapsto\left(\frac{\mathrm{e}^{d(t)} x}{\left(1+t^{n} y^{m}\right)^{1 / m}}, \frac{\mathrm{e}^{d(t)} y}{\left(1+t^{n} y^{m}\right)^{1 / m}}\right), \quad d(t)=1 / 2 \int c(t) d t .
$$




\section{ACKNOWLEDGMENT}

I. Cruz would like to thank Dr. John Rawnsley for all the help during the four year stay at Warwick and Dr. Mario Micalleff for some helpful remarks. Also the conversations with Professor Michel Cahen in Belgium were very much appreciated.

The work of J. Basto-Gonçalves was done while on leave at Instituto de Ciências Matemáticas de S. Carlos, USP-SC. Its hospitality is very thankfully acknowledged.

\section{REFERENCES}

[1] V. Arnold. Geometrical Methods in the Theory of Ordinary Differential Equations. SpringerVerlag, 1983. MR 84d:58023

[2] J. Basto-Gonçalves, I. Cruz. Analytic k-linearizability of some resonant Poisson structures. Letters in Math. Physics, 49, 1 (1999), 59-66. CMP 2000:04

[3] A. Brjuno. Analytical form of differential equations. Trans. Moscow Math. Soc., 25 (1971),131288. MR 51:13365]

[4] I. Cruz. The Local Structure of Poisson Manifolds. Ph. D. thesis, Warwick (U.K.), 1995.

[5] J. Dieudonné. Foundations of Modern Analysis. Academic Press, 1960. MR 22:11074

[6] J.P. Dufour. Linéarisation de certaines structures de Poisson. Journal of Differential Geometry, 32:415-428, 1990. MR 91m:58139

[7] E. Hille. Ordinary Differential Equations in the Complex Domain. Wiley-Interscience, 1976. MR 58:17266

[8] R. Roussarie. Modèles locaux de champs et de formes. Astérisque, 30, 1975. MR 55:13444

[9] S. Sternberg. On the structure of local homeomorphisms of Euclidean space II. American Journal of Mathematics, 80 (1958), 623-631. MR 20:3336

Departamento de Matemática Aplicada, Centro de Matemática Aplicada da Universidade do Porto, R. Das Taipas, 135, 4050-600 Porto, Portugal

E-mail address: jbgoncal@fc.up.pt

Departamento de Matemática Aplicada, Centro de Matemática Aplicada da UniverSidade do Porto, R. Das Taipas, 135, 4050-600 Porto, Portugal

E-mail address: imcruz@fc.up.pt 